\title{
NEOPOPULISMO DE LA FELICIDAD
}

\section{Beatriz Sarlo}

\|S ostenemos que estas verdades son autoevidentes, que todos los hombres son creados iguales, que están provistos por su Creador de ciertos derechos inalienables, que dentro de esos están la vida, la libertad y la búsqueda de felicidad."1 Esta declaración, escrita por Jefferson, trece años antes que la de la Revolución Francesa sobre los derechos del hombre y del ciudadano, nos introduce, desde el mismo comienzo, en una de las discusiones fundamentales de la política moderna. Una y otra vez, a lo largo de 250 años, los intereses y las ideas chocaron a propósito de una cuestión que aún hoy sigue abierta: ¿cuáles son los deberes de la política en este campo donde solo la libertad y la seguridad son considerados equiparables a la prosecución de la felicidad? Al mencionarlos explícitamente, la Declaración de la Independencia establece su equivalencia como derechos fundamentales que sostienen la legitimidad moral y política. Dejo de lado la discusión

Beatriz Sarlo es ensayista y escritora argentina. Ha sido fellow del Wilson Center (Washington), Simón Bolívar Professor of Latin American Studies en la Universidad de Cambridge y becaria del Wissenschaftskolleg, Berlín. Es autora del ya clásico Borges, un escritor en las orillas (1993). Algunos de sus últimos libros son Ficciones argentinas (Buenos Aires: Mardulce, 2012); Viajes: De la Amazonia a Malvinas (Buenos Aires: Seix Barral, 2014); Zona Saer (Santiago: UDP, 2016); La intimidad pública (Buenos Aires: Seix Barral, 2018) y es coautora de La lengua en disputa. Un debate sobre el lenguaje inclusivo (Buenos Aires: Ediciones Godot, 2019). Email: beatriz.sarlo@gmail.com.

1 "We hold these truths to be self-evident, that all men are created equal, that they are endowed by their Creator with certain unalienable Rights, that among these are Life, Liberty and the pursuit of Happiness." 
sobre si estos derechos tienen, a su vez, un fundamento trascendente, porque ello no afecta su equivalencia.

Persisten, en cambio, los debates de ideas y los conflictos políticos sobre la respuesta que se dé a la pregunta que define el lugar del Estado: aceptados los derechos como universales, ¿qué debe hacer el Estado para garantizarlos?, ¿cuál es la medida de una intervención justa?, ¿es suficiente la igualdad ante la ley o es necesario que esa igualdad formal se realice en estrategias que la vuelvan material y socialmente posible?, ¿compete al Estado reparar las diferencias que separan a los hombres de un ejercicio pleno de sus derechos?

No intentaré contestar estas preguntas, que desbordan mis posibilidades, ya que el debate comenzó al mismo tiempo que la declaración que instituía a la Felicidad en el lugar de los tres derechos fundamentales, abriendo así una larga tradición de igualitarismo democrático y una no menos larga y contenciosa tradición de republicanismo que rechazaba cualquier intervención del gobierno en el mercado que aspirara a una proporcionalidad en la distribución de los bienes y, por tanto, en las circunstancias en que los derechos se ejercen.

El debate, que comienza con Madison y los federalistas de un lado, y con Thomas Paine del otro, con diferentes figuras, algunas de ellas monstruosas, sigue hasta nuestros días. Como lo sintetiza Benjamin Radcliff $(2013,592,597)$ :

Fundamentalmente, Madison tiene como primer principio la noción de que el Estado debe respetar el derecho de quienes tienen riqueza a asegurar su propiedad, mientras que Paine cree que este derecho debe ser interpretado a la luz de sus efectos en aquellos que carecen de propiedad (...) Este es el corazón del desacuerdo y la base última de las diferencias que existen hoy entre derecha e izquierda. ¿Vemos al Estado con una obligación de proteger la riqueza privada (o, en el léxico de la derecha, 'libertad') o proveyendo 'felicidad general'??

\footnotetext{
2 "More fundamentally, Madison has as a first principle the notion that the state must respect the right of the wealthy to be secure in their property, whereas Paine believes that this right must be interpreted in light of its effects on those who lack property (...) This is the heart of their disagreement and the ultimate basis for the differences between the Right and the Left that exist today. Do we view the state as having an obligation more to protect private wealth (or, in the Lexicon of the Right, 'Liberty') or to provide for the 'general happiness'?’
} 
El Estado de Bienestar de la posguerra europea es uno de los capítulos de ese debate, que encontró sus réplicas en algunos regímenes latinoamericanos, especialmente en el primer peronismo, de 1945 a 1955, constructor de lo que he llamado 'Estado de Bienestar a la criolla', por las desprolijidades gigantescas, el personalismo y el populismo como ideología de sustento de democracias no liberales, con fuertes apoyos corporativos.

\section{LOS POPULISMOS MODERNOS}

En Argentina, el populismo es un rasgo tradicionalmente atribuido a gobiernos o partidos con tendencias nacionalistas, centralizantes y con escasa vocación institucional. En términos generales, tendencias no republicanas, pero sí democrático-plebiscitarias. El espacio simbólico populista es la Plaza donde se exhibe el Movimiento ante el Líder. Ambos se contraponen a la máquina de los partidos y las instituciones. No se trata sencillamente, como afirma Ernesto Laclau (2016), del establecimiento de dos campos ideológicos y políticos, sino de los diferentes instrumentos de acción en cada uno de esos campos y, sobre todo, de sus muy diferentes manifestaciones culturales y simbólicas, que definen un conjunto de estilos populistas que sostienen su capacidad hegemónica. No hay un populismo, sino diferentes manifestaciones históricas de las relaciones culturales entre política, dirigencias y Estado.

En primer lugar, sería inexacto afirmar que el populismo ha sido, en Argentina, siempre e invariablemente antirrepublicano. A comienzos del siglo XX, la Unión Cívica Radical, dirigida por Hipólito Yrigoyen, lleva adelante un largo conflicto con las elites políticas hasta obtener una ley fundamental para la democracia institucional: la de sufragio universal masculino, con voto secreto y obligatorio. Esa ley lleva a Yrigoyen a la presidencia en 1916. Todo puede decirse de este dirigente menos que careciera de elementos populistas en las formas de organización de su partido, la relación establecida con los nuevos votantes y el discurso mismo (carismático y misional) con que difunde sus ideas y construye la primera victoria electoral democrática.

La Unión Cívica Radical impone un cambio profundo en las costumbres políticas oligárquicas, que tampoco carecían de rasgos populistas. Lo que cambia es la forma de relación con bases sociales que, hasta 
comienzos del siglo XX, estaban excluidas de la decisión política o incluidas en estrategias de tipo únicamente clientelar y paternalista. Yrigoyen interpela a quienes hasta ese momento o no votaban o votaban siguiendo las instrucciones de sus patrones y caudillos. Votar a Yrigoyen representó una insurrección electoral, preparada en redes políticas de nuevo tipo.

Veinte años después, derrocado Yrigoyen por un golpe militar en 1930, un grupo de intelectuales y políticos, muchos de ellos radicales, fundaron FORJA, cuyo nombre mismo enuncia la orientación renovadora y nacionalista de un populismo de inspiración criolla: fuerza orientadora de la juventud argentina. En el campo cultural, FORJA es contemporánea de las diversas corrientes conocidas como revisionismo histórico, cuya diversidad, por izquierda y por derecha, no fue obstáculo para que coincidieran en un sistema de oposiciones que, desde entonces, ha tenido gran éxito en las versiones más populares del pasado argentino: litoral/interior; ciudad puerto/provincias; doctores y letrados/ Pueblo; patria/europeísmo.

Estos pares de conceptos antagónicos expresan, por una parte, la tensión entre elites letradas y masas populares; por la otra, la condición periférica y dependiente de la Argentina; y, finalmente, su constitución como país donde las regiones más alejadas del litoral atlántico sufrieron la opresión económica de las ciudades-puerto, especialmente de Buenos Aires. Tales oposiciones, aunque hoy no formen parte central del conocimiento producido por la historia académica, siguieron siendo ideológicamente productivas; fueron la ideología del gobierno kirchnerista, que le dio al revisionismo histórico y sus sacerdotes un lugar destacado, convirtiéndolo en la espontaneidad del discurso presidencial de Cristina Kirchner.

Pero antes del kirchnerismo, el mercado editorial ya había descubierto que libros y revistas que trasmitieran el relato revisionista se convertían en éxitos de venta por varias razones: evitaban la pesadez argumentativa y probatoria de la historia académica; daban una versión dramatizable de un pasado complejo, es decir: una historia popular no solo por sus contenidos sino por su forma narrativa; y sus autores, precisamente porque no eran académicos, manejaban las destrezas del periodismo. Las oposiciones (Nación vs Imperio, Pueblo vs elites) que organizaban la historia revisionista sintonizan perfectamente con un 
relato donde amigos y enemigos eran definidos con claridad y ya le habían transferido al sentido común las claves de una historia que ofrecía la ventaja de prolongarse en una narración que anuncia e interpreta el presente. A esta retórica de la ideología la he llamado populismo histórico de mercado. Ofrece una versión sencilla y convincente de los corsi e ricorsi del pasado nacional. A medida que la historia académica alcanza sus mejores niveles, el revisionismo de mercado la supera en lectores e instruye una Doxa. ${ }^{3}$

Bronislaw Baczko $(1991,21)$ afirma:

Uno de los caracteres fundamentales del hecho social es precisamente su aspecto simbólico. En la mayor parte de las representaciones colectivas no se trata de una representación única, de una cosa única, sino de una representación elegida más o menos arbitrariamente para significar otras y para impulsar prácticas.

La construcción de un relato histórico ha sido, hasta hoy, un capítulo de la construcción de identidades colectivas. En el caso argentino, el peronismo fue, a partir de la década de 1960, un objeto privilegiado por los discursos de intención histórica e identitaria.

Sucesivas oleadas de interpretación del peronismo forman parte de un capítulo extenso de historia intelectual y de historia política argentina. Las variaciones interpretativas fueron desde la matriz marxista con acentos nacionalistas y trotskistas, que definió al peronismo como bonapartismo, hasta la marxista antiimperialista, que lo consideró la forma culminante del nacionalismo democrático de masas en una nación dependiente. Ernesto Laclau (2016) fue el último en intentar esta aventura filosófica. Su populismo define a una identidad parcial, el Pueblo, como única identidad total legítima. Y requiere la división de la sociedad en dos campos, uno de los cuales se presenta como parte que reclama ser el Todo.

Con todas estas variantes historiográficas y filosóficas, el populismo argentino, identificado generalmente con el peronismo o con una línea de ese movimiento, apareció como única dimensión ideológica capaz de producir fuertes identificaciones simbólicas y por lo tanto identidades políticas sostenidas en identidades culturales. Sería la articula-

${ }^{3}$ Sobre revisionismo, véase Quattrocchi-Woisson (1995). 
ción de masas movilizadas y de un líder carismático, en una escena de democracia plebiscitaria con régimen no republicano liberal. Este fue el populismo del kirchnerismo, no tanto o no solo por influencia de Laclau (alguien demasiado arduo para leer salvo por intelectuales), sino por una vulgata que toma hilos de todos los populismos anteriores y que, sobre todo, tiene conciencia política de que para prevalecer es indispensable definir un Enemigo (interno, externo, amenazante o vencido, real o fantasmático). Algo así como un movimiento schmittiano, cuyos dirigentes kirchneristas muy probablemente no habían leído a Carl Schmitt, como lo leyeron y aplicaron Laclau y, de modo más articulado, Chantal Mouffe. Si se admite una nota filológica: Arturo Sampay, un intelectual importante del primer peronismo, conocía a Schmitt (Dotti 2000).

Tales fueron, en la Argentina, los 'populismos modernos'. Influyeron en el debate intelectual y en la práctica política a la que le ofrecieron un vocabulario común para la interlocución entre dirigentes y masas. Tradujeron de un dialecto social a otro, produjeron una lengua común. Taguieff (1996) subraya que el nacional-populismo presupone no solo al Pueblo como sujeto sino a un Líder que sea capaz de constituirlo mediante una interpelación exitosa. Por eso, agrega, "el partido populista debe ser hiperbólicamente personalizado" (Taguieff 1996, 56-59). Y el Pueblo que vive en el discurso es convocado para una tarea redentora de sí mismo y de la Patria.

\section{FORMAS ACTUALES: LOS POPULISMOS POSMODERNOS}

Este tipo de discurso, que llamo 'populismo moderno', puede eventualmente volver a tener un lugar en la esfera política; ya en el siglo XXI, el kirchnerismo argentino presentó, entre otros rasgos, la capacidad de movilización cultural e ideológica que se atribuye a los liderazgos carismáticos. Pero el paisaje actual tiene los colores de lo que llamaría los populismos posmodernos, sin hacer de ese adjetivo una llave para resolver todos los enigmas, sino un indicador que caracteriza configuraciones culturales diferentes de las conocidas en Occidente en el siglo pasado.

La configuración neopopulista es propia de sociedades de masas emancipadas de las culturas tradicionales, es decir, sociedades constituidas por el mercado y por la cultura mediática industrial y sus de- 
rivaciones. Allí, al parecer, se han debilitado identidades ciudadanas ya adquiridas (por ejemplo, en el mercado de trabajo y la educación formal). Diferente de los populismos cultural-políticos organizados por el eje Pueblo-Líder y apoyados con frecuencia en organizaciones como los sindicatos, surge un populismo de la 'sociedad' o populismo de la 'gente', que es en realidad un populismo del mercado.

Algunos ejemplos: el populismo juvenilista (que coloniza desde el mercado hasta el discurso educativo); el populismo estético, desde el pop, pero acentuadamente hoy por la pregnancia de la iconografía popular en las artes plásticas; el gusto letrado por lo kitsch, que comienza con la relectura 'culta' de la telenovela y, poco más tarde, el reality; el populismo de una opinión pública alejada de la esfera política y de los partidos que se expande en las redes sociales.

El análisis cultural latinoamericano, especialmente en la obra de Jesús Martín Barbero (2010) (alguien que recibió tempranamente las tesis de Michel de Certeau), desde hace décadas describe al consumidor de los bienes del mercado como alguien que no tiene límites para rearmar lo que recibe. En contra de las teorías del determinismo del mensaje, esta teoría de la recepción sostiene la libertad semiótica del espectador. Su tono es generalmente optimista y opuesto a las versiones catastrofistas en las que los medios definen y conforman identidades. En la caracterización de 'consumidores' y 'ciudadanos' que realizó Néstor García Canclini (1995), las prácticas de los ciudadanos frente al mercado distanciaban a esos 'consumidores' de la identificación que quisieron imponer las elites: un pueblo de ciudadanos. Abundaron los discursos celebratorios. Sin embargo, lo que tuvo lugar fue una crisis, de la que el mercado salió victorioso y con capacidad para diseñar inclusiones y exclusiones decisivas. Esa crisis afectó a los sistemas educativos en una dirección que contribuyó a las desigualdades. Afectó también la legitimidad de las elites letradas, ya que las redes sociales se caracterizan por una horizontalidad sustentada en valores que desplazan a los emisores tradicionales, sean estos de 'izquierda' o de 'derecha' (lo escribo con las comillas como supersigno de otro rasgo de la posmodernidad: el fin llamado de las ideologías).

La pérdida de densidad del discurso político tiene una relación continua, aunque inestable, con la influencia de los medios y de la encuestología sobre la política. Si se habla de los medios y se leen las 
encuestas, lo que se construye ya no es el pueblo sino 'la gente'; el político pierde su apellido y se lo designa por su nombre de pila: Mauricio, Gaby, Cristina, Evo, lo que, salvo en Brasil, es una novedad. Solo unos pocos conservaron su apellido y, en cada caso, la explicación debe buscarse en particularidades políticas nacionales: Bachelet, Lagos, Chávez, Correas. El nombre de pila es la forma de representar una proximidad imaginaria. Los políticos, como las estrellas mediáticas, necesitan ser vistos como entes cercanos, aunque un subtexto indique que son excepcionales e inalcanzables.

La proximidad de los políticos neopopulistas con estas estrellas y su intensa y nerviosa relación con los medios y las redes se retroalimentan, como lo señala Mario Riorda $(2016,7)$ refiriéndose a Macri:

\begin{abstract}
Más que a los medios tradicionales, este gobierno les presta muchísima atención a los actores con incidencia en los medios. Las reuniones de Macri con Mirtha Legrand y con Marcelo Tinelli son ejemplos notables de que a todo aquello que incide le presta atención (...) Me parece que con la mayoría de los principales medios de Argentina, este gobierno tiene una buena relación y hasta incluso delegativa, en tanto permite que los medios editorialicen del gobierno muchas cosas que el propio gobierno no editorializa de sí mismo. Terceriza parte de la construcción de su mito de gobierno en actores con pluma propia.
\end{abstract}

Estos cambios en la esfera simbólica tienen lugar en condiciones económicas desconocidas hasta los años ochenta del siglo pasado, impuestas por una pobreza de nuevo tipo, que destruyó anteriores lazos comunitarios, barriales, espaciales y familiares. Hoy, hombres y mujeres no están en condiciones materiales de pensarse como integrantes de una nación que, a su vez, integra. Este fue tanto el mito como el impulso real de buena parte del siglo XX en Argentina. A comienzos del XXI, predominan los efectos de una amenaza que, para un tercio de la población, se ha vuelto demasiado real: la caída y la pérdida.

Las sucesivas crisis económicas deformaron el paisaje que se creía conocer. Argentina llegaba al fin del siglo XX convertida en un territorio de exclusión. Nos 'latinoamericanizábamos', para decirlo con el verbo que le daba nombre al aumento de la pobreza y la desintegración económico-social. Durante 2001, año de alerta y desenlace, las 
ideas sobre Argentina cambiaron quizás tanto como había cambiado la realidad. Cuando el cambio ya no es novedoso para nadie, quedan sus consecuencias solo parcialmente compensadas por políticas sociales. Quienes no cayeron en la pobreza saben, aunque borrosamente, que un tercio del país ha caído. El paisaje de la pobreza es ineliminable. De todos modos, este saber se entrelaza, de modo imaginario y real, con las posibilidades de consumo de los 'salvados'. Para ellos, nada hay más persuasivo que el consumo y el mercado.

El resultado es la desagregación sociocultural que toca a cerca de un $30 \%$ de la población. ${ }^{4}$ Hoy, más que nunca puede hablarse de un proceso que va en sentido opuesto a las fuerzas que caracterizaron la sociedad argentina hasta mediados del siglo XX. Los conflictos ideológicos de hace cuarenta años fueron enconados y no incorporaban ninguna posibilidad de resolución que evitara la escalada de violencia ni su trágico cierre con el terrorismo de estado. Sin embargo, las cosas sucedían en una sociedad relativamente integrada en términos sociales, producto de las décadas anteriores, cuyo impulso se mantuvo hasta comienzos de la década de 1980.

En primer lugar, estuvo la integración en la escuela pública, gratuita y obligatoria para niñas y niños; luego, de manera sobresaliente, la integración en el mercado de trabajo y las experiencias de representación sindical en ese espacio y en la esfera pública. El mundo popular tenía formas de representación generalizadas, aunque ellas fueran de calidad desigual y sobre todo alejadas de un funcionamiento democrático pleno. Esas formas transcurrían, en una dimensión, a través de partidos políticos, con ideologías hegemonizadas a partir de los años cincuenta por la persistencia de la identidad justicialista; pero, también, y fundamentalmente, por diversos niveles de representación sindical, asociativa y territorial. No había un vacío de referencias, sino más bien un pleno identitario, que las organizaciones de izquierda consideraban un obstáculo organizativo y simbólico a su expansión.

En segundo lugar, se produjo un giro en el tipo y extensión de la hegemonía mediática. La hegemonía audiovisual fue acompañada por el descenso en la lectura de diarios, y esto le dio un tono particular a la cultura pública y a la política. La televisión se impuso como construc-

${ }^{4}$ INDEC y Observatorio de la Deuda Social de Argentina-Universidad Católica Argentina. 
tora de una esfera pública donde la competencia electoral y la confrontación de ideas eran procesadas en torneos de popularidad mediática. A este perfil comunicacional, las redes sociales aportaron su promesa democratista que no se cumple invariablemente o, más bien, que se cumple solo en condiciones muy precisas: las redes pueden llamar a una manifestación, pero es bien difícil sostener, en las redes, la continuidad temporal y la complejidad argumentativa de la política. Rosanvallon (2011) se refiere a este punto:

Esta opinión borrosa y confusa que existe a través de Internet, porque Internet no es un medio, sino una forma social, una especie de materialidad directa y conmovedora de una opinión pública que antes solo existía representada por las instituciones, los medios de comunicación y las técnicas de encuesta. ${ }^{5}$

Si el teórico francés es relativamente optimista, de todos modos, imposible pasar por alto que el espacio público, la escena simbólica de la nación, está fracturado en secciones diferenciadas social y culturalmente hasta el detalle. Aquello que los teóricos del mercado denominan 'nichos' culturales, son verdaderos agujeros de segregación y producción de diferencias. En estas condiciones es casi ilusorio hablar de un espacio público nacional. Basta consultar los datos educativos de Argentina para definir clivajes y fracturas que tienen consecuencias de largo plazo, en una sociedad desarticulada en tribus sin solidaridades, que se agrupan por pulsiones. A diferencia del melodrama romántico y moderno, el nuevo melodrama social carece de lugares e indicaciones estabilizadas. Y esto repercute en identidades estalladas, que no se reconocen en principios generales, sino en parcialidades culturales. De allí la importancia del deporte y de la música, identificaciones resistentes a las que sería equivocado considerar fugaces. Hoy, la nación deportiva es el único gran imaginario transclase: el fútbol, una luz enceguecedora, cuyo dínamo está en el mercado.

\footnotetext{
5 “Cette opinion indistincte et confuse qui existe à travers Internet, car Internet n'est pas un média, mais une forme sociale, sorte de matérialité directe et mouvante d'une opinion publique qui n'existait autrefois que représentée par des institutions, des médias, des techniques de sondage."
} 


\section{POPULISMO DE LA FELICIDAD}

No es casual que Mauricio Macri venga del fútbol. Presidente de Boca Juniors, ese fue su primer cargo electivo. En un cursus honorum abreviado si se lo compara con el de cualquier político argentino con militancia en los partidos o los sindicatos, de allí pasó, directamente, a la jefatura de gobierno de la ciudad de Buenos Aires. Y de allí a la presidencia de la república. No fue dirigente estudiantil, ni gremial, ni militar, ni partidario, ni participó en otras actividades que las del fútbol y el empresariado (aunque tampoco en esta esfera fue dirigente). Como Donald Trump, entró en la política porque era rico, sin que su riqueza fuera complementada por un pasado de ascensos, en general largos y trabajosos. Representa una nueva forma de llegada, que vacilaría en llamar excepcional, ya que puede ser la primera de una serie de nuevo tipo.

A diferencia de Alfonsín o Néstor y Cristina Kirchner, Macri carece de un relato histórico que dé razón de su propio pasado y lo enlace con el de la República o el de la Nación. En su convocatoria, "los valores 'morales', 'tradicionales', 'naturales' o del 'sentido común' sustituyen a la ideología, a fin de alcanzar la unidad interclasista" (Taguieff 1996, 55). Carece de una epopeya o mito fundacional, en la medida en que es imposible convertir un club de fútbol, por mayor renombre mundial que posea, en una larga marcha hacia el poder, a lo Mussolini; o en una insurrección popular, como el 17 de octubre, donde el Pueblo rescató a su Líder, exigió su libertad y, finalmente, lo puso en la casa de gobierno. ${ }^{6}$

En épocas todavía cercanas, Chávez también protagonizó una larga marcha que incluyó elecciones, desplazamientos, prisiones, golpes de estado, regresos. E incluso Néstor Kirchner, cuando asumió como presidente de Argentina, evocó una marcha que se extendía desde los años de la juventud peronista con sus ideales nacionales y revolucionarios (que, como dijo en su primer discurso, no estaba dispuesto a dejar a las

\footnotetext{
${ }^{6}$ Mussolini, por ejemplo, antes de la marcha sobre Roma, pronuncia un discurso que culmina así: "El momento es para nosotros propicio; es más, diría venturoso. Si el gobierno hubiese de ser inteligente, nos dará el poder pacíficamente; si no hubiese de ser inteligente, lo tomaremos por la fuerza. Debemos marchar sobre Roma para quitársela de las manos a los politicastros pusilánimes e ineptos. Cuando suene la campana, marcharemos como un solo hombre" (discurso de agosto de 1922, cit. en Gentile 2014, 151).
} 
puertas de la casa de gobierno) hasta el presente. Con alto valor simbólico, el día de su investidura, el nuevo presidente se bajó de su automóvil, se acercó a la multitud, estrechó manos, fue abrazado e, incluso, se lastimó la frente con la cámara de un fotógrafo demasiado ansioso. Realizó los gestos y pronunció las palabras adecuadas a lo que Sigal y Verón (1985) llaman el 'paradigma de la llegada': "vengo desde lejos", dijo Perón, cuando regresó a la Argentina después de 18 años de proscripción. "Vengo de la clase obrera" se escuchaba, de diversos modos, en la primera etapa de los discursos de Lula como candidato.

Macri, gran cambio, llega al gobierno de la ciudad de Buenos Aires desde Boca Juniors. Y el día de su asunción como presidente, al salir al histórico balcón de la casa de gobierno, se sacó la banda azul y blanca, símbolo nacional centenario, para bailar más cómodamente frente a quienes lo miraban desde la Plaza de Mayo. A diferencia de los dirigentes calientes, su epopeya fundacional es cool.

Frente a la ausencia de alternativas sociopolíticas que puedan despertar el entusiasmo, Macri se ajusta a las leyes de una economía simbólica de la felicidad, donde ese concepto es usado en sus acepciones vulgares, sin rastros del debate de 250 años que se mencionó al comienzo. Durante la campaña electoral que lo llevó a la presidencia, los asesores de discurso de Macri sostuvieron esta estrategia discursiva con una convicción tan fuerte como vacía de contenidos sustanciales. Según el ideólogo de esa campaña, citado por uno de los que trabajaron en ella: "Nosotros no buscamos votos, buscamos que nuestros candidatos tengan buena imagen; si tenés buena imagen, los votos van a venir" (Iglesias 2016, 59). Buena imagen es eso, brutalmente dicho: no mostrar nada sino aquello que sintonice con lo que funciona probadamente en los medios y las redes sociales; hablar lo menos posible, poner en escena una proximidad ilusoria con la gente, tocar y ser tocado, mirar y ser visto.

Esa fabricación de la proximidad ha tenido, hace muy poco, dos escenas culminantes. La primera: un viaje de Macri en colectivo (como se designa en Argentina a los ómnibus urbanos), para el cual se montó una filmación y se hizo un casting, como si se tratara de un film publicitario, aunque el gobierno lo difundió como 'salida espontánea'. La segunda: incorporar a su hija de cuatro años a las visitas dominicales que, acompañado por fotógrafos, realiza a los habitantes de casas en 
barrios populares, pero no villas miseria, en los distritos del conurbano. Un neopopulismo no solo cool sino proclive a la manipulación.

Mientras se insiste en estas acciones de mínima sustancia política, se leen encuestas cuantitativas y se hacen focus groups, con el fin de descubrir en esas fuentes, como dijo un asesor antes de que Macri llegara a la presidencia, "cosas que son populares y son buenas para el país. Después vemos cuando seamos gobierno cómo hacemos para conseguirlo" (Iglesias 2016, 229). Desde una perspectiva clásica se podrá objetar la carga de improvisación encuestológica. Pero, desde la perspectiva del nuevo populismo, la ausencia de convicciones políticas de los dirigentes es más bien el indispensable rasgo positivo de la nueva situación, donde se compite por el apoyo de la 'gente', a la que se define movilizada por las mismas valencias que las movilizan en el mercado. La política ha perdido su especificidad discursiva y práctica.

El neopopulismo decidió su consigna de campaña del mismo modo. La consigna fue Pobreza Cero, aunque el candidato presidencial (que es un orador menos que mediocre) tuvo dificultades en exponerla, entre otras razones porque es un horizonte utópico en el que probablemente se sienta incómodo. Durante la campaña por la presidencia, el equipo de discurso fue todopoderoso, tal como lo explica uno de sus integrantes: "Esta mañana estuvimos a punto de cambiar el nombre de 'Pobreza Cero' por el de 'Un país de clase media' o algunas de sus variantes" (Iglesias 2016, 230). Quien sugiere el cambio, lo hace porque Pobreza Cero presenta el problema de ser una consigna negativa, "mientras que la referencia a la clase media permitía a los electores identificarse con un sueño". Sin embargo, quedó Pobreza Cero porque la consigna que prometía el ascenso a la clase media sonaba paternalista y además colocaba un 'techo', un 'hasta aquí nomás'.

Cualquiera que haya formado parte de un equipo de campaña política (y ese es mi caso) sabe que estas discusiones de mercadotecnia son casi inevitables. Sin embargo, la particularidad del equipo de Macri fue (y sigue siendo) que estas discusiones desplazan por completo cualquier otra que tuviera como objeto reintroducir la política en el discurso presidencial. Una y otra vez, el equipo de discurso se confirma en la idea de que no hay que hablar de nada o de que hay que hablar de la manera menos concreta posible. Se diseña así un modelo donde el momento imaginativo y creador de la política es considerado un lastre pretérito. 
O peor aún: se considera que el programa con el que se gobernará en caso de ganar elecciones, no debe ser conocido por los votantes que se aburren con esas cosas. ¿Porque se aburren? ¿Acaso transmitir claramente las medidas de gobierno que pueden afectar sus vidas no es un deber ético del político? Esa sería una interpretación benevolente del discurso de la felicidad sin sustancia. También podría pensarse en otras dos razones: la primera, que ese programa no existe del todo y que, por eso, quedará librado a un juego conflictivo de presiones e intereses; la segunda, porque ese programa es inconfesable y contradictorio con las consignas 'pobreza cero' o 'un país de capas medias'.

En consecuencia, el neopopulismo tiene la obligación del seguidismo a una opinión construida en las encuestas, como si esa construcción fuera, en primer lugar, una verdad de fuerte valor empírico (cosa que está por verse según más cuidadosos exámenes de método) y, en caso de serlo, como si esa base empírica fuera el techo y no el piso de la creación política. Viene al caso la crítica ya clásica de J.-C. Passeron al populismo, "para quien el sentido de las prácticas populares se cumple íntegramente en la felicidad monádica de la autosuficiencia simbólica" (Grignon y Passeron 1989, 31). Hoy diría, el 'culto de la proximidad manufacturada'.

De tal modo, la promesa de felicidad no abre un debate, como lo abrió cuando Jefferson inscribió ese derecho entre los tres fundamentales, sino que lo cierra, dado que la política no se hace cargo del conflicto implícito: si la consecución de la felicidad es un derecho ¿debe proveerse a ese derecho o simplemente enunciarlo sin producir las condiciones materiales para que se convierta en realidad social? El neopopulismo no desea ni está en condiciones de enfrentar esta pregunta, ni desea considerar valores centrales como equidad, igualdad de oportunidades, etc., etc.

La felicidad vuelve al discurso político como un resto degradado, ocasional, cuando una tendencia neopopulista cree descubrir allí no un problema sino una consigna. El neopopulismo cree que algunos conceptos son una máquina de licuar conflictos que no se extienden solamente a lo simbólico. O, como lo fraseó en sus instrucciones de campaña electoral el maestro de mercadotecnia elegido: "Mauricio [Macri] venía menos a proponer un cambio que a acompañar y representar el deseo de cambio que ya estaba en la gente" (Grignon y Passeron 1989, 22). 
Imposible solicitar que este neopopulismo tome en consideración que existe el conflicto entre el deseo individual de felicidad y la trama social donde se enfrentan esos deseos en sus diferencias e incompatibilidades. El neopopulismo considera que este tema es ideológico y que las ideologías han muerto. O, como lo frasea el jefe de gabinete el 15 de junio pasado, en Twitter: "Juntos vamos a lograr lo que parece imposible". Ni un paso atrás ni uno adelante: "Todo es posible juntos", como instruye una de las consignas que hoy repite el gobierno.

Palabras de Macri en un discurso del 19 de julio de 2015:

Creo en una política distinta. Una política que despierte a la gente en lugar de adormecerla. Que saque lo mejor de cada uno. Que aspire a potenciar la vida y la esperanza, no que fomente el miedo y la resignación. Creo en la solidaridad y en el trabajo en equipo. En una sociedad donde las personas entiendan a su vecino como alguien con quien compartir y construir en conjunto, respetando sus diferencias. Creo en la honestidad y en la humildad de los argentinos. No admito la idea que los argentinos somos corruptos y mentirosos. Somos mejores que lo que han hecho de nosotros las equivocaciones de muchos de nuestros dirigentes.

¿Qué decir? La sincera obviedad de los temas vuelve superfluo cualquier análisis. Sin embargo, algunos temas ideológicos merecen un subrayado. ${ }^{7}$ El primero, que tiene ecos de nacionalismo individualista, sostiene que los argentinos somos mejores que lo que han hecho de nosotros los errores de los políticos. El tema es el de la reconciliación populista con una autoimagen fantasiosa, que pasa por alto la afirmación opuesta: que los argentinos son peores que algunos de sus dirigentes (como se demostró en la indiferencia de los argentinos frente a la dictadura militar, que solo tuvo como opositores a algunos dirigentes de movimientos sociales y unos pocos políticos; o la indiferencia de los argentinos frente a los procesos de pauperización de la década de 1990, porque las capas medias no se sentían directamente amenazadas). El tema resuena con la antigua contraposición populista entre gente del común y elites. Y su función es salvadora. Este tema se enlaza con el

\footnotetext{
${ }^{7}$ Estos temas pueden leerse en los materiales de la web de Mauricio Macri: http://pro. com.ar; y en las cuentas de Twitter y Facebook.
} 
de la 'honestidad y la humildad de los argentinos': dos cualidades que es difícil reconocer al unísono. La honestidad tiene algunas formas de medirse, entre ellas las de la evasión impositiva, el trabajo en negro, la precariedad laboral, el intercambio de coimas y favores, que diferencian muy claramente las costumbres argentinas de las de sus vecinos de Chile y Uruguay.

Esta visión complaciente de los argentinos es indispensable en el discurso neopopulista, ya que este no propone una reforma intelectual y moral que sus potenciales votantes probablemente no estén dispuestos a aceptar como tarea pendiente. El neopopulismo, como el paleo-populismo, necesita embellecer el Pueblo (en general capas medias) al que aspira a convertir en su clientela política gratuita. Así se protege una identidad mitologizada. Por cierto, este pueblo supuestamente 'honesto y humilde' merece la felicidad como recompensa a esas virtudes que fueron traicionadas por políticos que carecieron de humildad y honestidad. De nuevo, en este caso una operación imaginaria que separa radicalmente a los gobernantes de los gobernados, olvidando los momentos más comprometedores de la historia argentina del último medio siglo. El neopopulismo de derecha se especializa en estas operaciones, de las que excluye tanto a la sociedad realmente existente como a sí mismo. Como es un populismo posmoderno, cool, de baja tensión, no se presenta como el populismo clásico. Por el contrario, abjura del cesarismo, el funcionamiento plebiscitario, la movilización y el carisma caliente de sus liderazgos. Tampoco confía en convocar fuerzas existentes en la sociedad y sus organizaciones. Con desnuda evidencia, trae un discurso que no lo obliga a compromisos con las fuerzas sociales. Afirma sencillamente que desea un imposible, pobreza cero, por ejemplo, como ha dicho Mauricio Macri.

Jugar con el imposible sin definirlo implica el juego de la ensoñación. Todos queremos ser felices. Para decirlo con palabras en las que coincidieron Hannah Arendt y Franklin Roosevelt, todos deseamos liberar nuestras vidas de las condiciones de necesidad y del miedo a caer en esas condiciones. Llegados a este punto, se reabre un viejo debate sobre sociedad y Estado: ¿hay algo que el Estado puede hacer en la prosecución de la felicidad? El discurso neopopulista sostiene, cuando habla en serio y no en función mediática, que el Estado debe garantizar la libertad. No cabe duda, pero la pregunta sigue abierta. Dos siglos de historia 
debatieron el quantum de lo que debe hacer el Estado. El neopopulismo de la felicidad se inclina, en el caso argentino, a que ese quantum sea programática e idealmente mínimo, excepto si debe enfrentar situaciones excepcionales de intranquilidad social. $i Y$ si el mercado produce una inseguridad incompatible con el mito de la felicidad? La respuesta a esta pregunta está en el centro del debate. La 'mano invisible' del mercado repartirá sus dones. El neopopulismo de la felicidad confía en hacer política en estado de baja politicidad. El concepto-mito correspondiente podría llamarse 'optimismo país'.

\section{BIBLIOGRAFÍA}

Baczko, B. 1991. Los imaginarios sociales. Memorias y esperanzas colectivas. Buenos Aires: Nueva Visión.

Dotti, J.E. 2000. Arturo E. Sampay: el 'schmittiano' que nunca fue tal (135-166). En Carl Schmitt en Argentina. Buenos Aires: Homo Sapiens.

García Canclini, E. 1995. Consumidores y ciudadanos. Conflictos multiculturales de la globalización. México DF: Grijalbo.

Gentile, E. 2014. El fascismo y la marcha sobre Roma. Buenos Aires: Edhasa.

Grignon, C. y Passeron, J.-C. 1989. Miserabilismo y populismo en sociología y en literatura. Buenos Aires: Nueva Visión.

Iglesias, H. 2016. Cambiamos. Mauricio Macri Presidente. Día a día, la campaña por dentro. Buenos Aires: Sudamericana.

INDEC, Instituto Nacional de Estadísticas y Censos 2019. Disponible en: www. indec.mecon.ar.

Jefferson, T. 1776. Declaration of Independence. Disponible en: www.archives. gov/exhibits/charters.

Laclau, E. 2016. La razón populista. México DF: Fondo de Cultura Económica.

Martín Barbero, J. 2010. De los medios a las mediaciones. Comunicación, cultura y hegemonía. Barcelona: Anthropos.

Observatorio de la Deuda Social de Argentina-Universidad Católica Argentina 2019. Disponible en: uca.edu.ar/es/observatorio-de-la-deuda-social-argentina.

Quattrocchi-Woisson, D. 1995. Los males de la memoria. Buenos Aires: Emecé.

Radcliff, B. 2013. The Political Economy of Human Happiness. New York: Cambridge University Press.

Riorda, M. 2016. Macri terceriza la construcción de su mito de gobierno en algunos medios. "Ideas", La Nación, 4 de septiembre, 7.

Rosanvallon, P. 2011. Penser le populisme. La Vie des idées. Disponible en: www. laviedesidees.fr/penser-le-populisme-html. 
Sigal, S. y Verón, E. 1985. Perón o muerte. Los fundamentos discursivos del fenómeno peronista. Buenos Aires: Legasa.

Taguieff, P.-A. 1996. Las ciencias políticas frente al populismo: de un espejismo conceptual a un problema real (56-59). En Adler, F., Fleming, T., Gottfried, P., Luke, T., Piccone, P., Taguieff, P.-A. y Wilson, C., Populismo posmoderno. Bernal: Universidad Nacional de Quilmes. EP 